\title{
Clinical Efficacy of the 940-nm Diode Laser in the Treatment of Recurrent Pockets in the Periodontal Maintenance Phase
}

\author{
Zahra Alizadeh Tabari $^{1}{ }^{\circledR}$, Leila pournasir ${ }^{2}$, Sadegh Mohammadreza ${ }^{3}$, Fahimeh Anbari ${ }^{* *}$ \\ ${ }^{1}$ Department of periodontics, school of dentistry, Qazvin university of medical sciences, Qazvin, Iran \\ ${ }^{2}$ Periodontist \\ ${ }^{3}$ post Graduate Student of Periodontics, School of Dentistry, Shahid Beheshti University of Medical Sciences, \\ Tehran, Iran \\ ${ }^{4}$ Oral Medicine Department, School of Dentistry, Shahid Beheshti University of Medical Sciences, Tehran, Iran
}

\section{*Correspondence to Fahimeh Anbari, Assistant professor, oral medicine department, school of dentistry, Shahid behest university of medical sciences, Tehran, Iran. Phone: +989203894001; Email: fahimeh.anbari@gmail.com}

Received: January 29, 2021 Accepted: June 27, 2021 Published online November 6 , 2021

\begin{abstract}
Introduction: The basis of periodontal treatments is the mechanical removal of bacterial biofilm, which is often not sufficient. Therefore, laser therapy can be effective as an adjunct treatment. The aim of the present study was to evaluate the clinical efficacy of the 940-nm diode laser in the treatment of recurrent pockets of patients in the periodontal maintenance phase.

Methods: The present clinical trial study was performed on 20 patients. Clinical indices, including bleeding index $(\mathrm{BI})$, probing pocket depth (PPD), and clinical attachment level (CAL) of the studied teeth were recorded before the test. The different quadrants were randomly divided into two experimental (scaling and root planing (SRP) + Laser) and control groups (SRP alone). Both groups were matched in terms of plaque index (PI). Clinical indices were re-recorded using therapeutic methods 30 and 90 days after the treatment, and data analysis was carried out using the $t$ test and LSD.

Results: There were no significant differences in PI, PPD, CAL, and $\mathrm{BI}$ in both groups before the treatment $(P>0.05)$. However, there were significant differences in PPD, CAL, and $\mathrm{BI}$ in the two groups one month after the treatment $(P<0.05)$; it means that improvements were more significant in the experimental group than in the control group. Comparing experimental and control groups, we found that there were no significant differences in PPD and CAL indices three months after the treatment $(P>0.05)$; however, there were significant improvements in the $\mathrm{BI}$ index in both groups $(P<0.05)$, so that the improvement was better in the experimental group than the control group.

Conclusion: Both SRP + Laser and SRP alone improved clinical indices in patients. In short-term follow-up, the effect of SRP+Laser treatment on BI, CAL, PPD was significantly superior to SRP treatment alone, but in long-term follow-up, SRP + laser was more effective than SRP alone in improving the $\mathrm{BI}$ index.

Keywords: Diode laser; recurrent pockets; Periodontal maintenance phase.
\end{abstract}

\section{Introduction}

Chronic periodontitis is a common inflammatory disease caused by bacteria in a susceptible host and leads to loss of supporting structures around the teeth. ${ }^{1}$

Various treatments, both surgical and non-surgical, have been suggested for the treatment of periodontitis. In addition to oral hygiene instruction, scaling and root planing (SRP) is the main non-surgical method for removing or reducing subgingival bacteria that stimulate gingival inflammation. ${ }^{2}$ However, limitations related to difficulty in accessing furcations, grooves, and deep pockets have led to the exploration of other therapeutic modalities. ${ }^{3}$ In fact, making definitive statements regarding the efficacy of treatment modalities based on available information is inappropriate. ${ }^{4}$

Periodontal surgery may be used to ensure better access to root surfaces in areas that do not respond to non-surgical treatment, such as teeth with infrabony or vertical defects. ${ }^{5,6}$ Clinical studies show that the long-term success of periodontal treatments depends on regular and continuous maintenance phases after the active treatment phase. ${ }^{7}$ During the periodontal maintenance phase, some areas may have a persistent infection or be reinfected. ${ }^{8}$ Residual pockets cause the persistent presence

Please cite this article as follows: Alizadeh Tabari Z, Pournasir L, Mohammadreza S, Anbari F. Clinical efficacy of the 940-nm diode laser in the treatment of recurrent pockets in the periodontal maintenance phase. / Lasers Med Sci. 2021;12:e68. doi:10.34172/jlms.2021.68. 
of periodontal pathogens ${ }^{9}$ and provide an environment for colonization of bacteria that are incompatible with periodontal health ${ }^{10}$ and may undergo frequent therapies; therefore, maintenance protocols must be not only efficient but also harmless. Since scaling may lead to the unnecessary removal of the tooth structure, frequent use of this method cannot be an ideal treatment option. ${ }^{11,12}$ Also, antibiotics cannot be an alternative because their repeated use can lead to problems such as bacterial resistance. Therefore, there is a need for careful evaluation of new treatment protocols that are both efficient and harmless so that they can remove bacterial biofilm without causing injury to the host tissues. ${ }^{13}$ One of these treatments is the application of laser energy. ${ }^{14}$ Lasers are used in dental plaque removal, bone surgery, soft tissue correction, gingivectomy, gingival curettage, and melanin pigmentation removal. ${ }^{15}$ Among the lasers, the diode laser is one of the most popular laser technologies considering its low price, portability, and ease of use. ${ }^{16}$

Most studies use a laser as an adjuvant treatment along with SRP versus SRP alone in the treatment of periodontitis in the early stage ${ }^{17-19}$ and there are few studies on the use of diode lasers to treat recurring pockets. Therefore, in this study, we investigated the clinical effects of a $940-\mathrm{nm}$ diode laser in the treatment of recurrent pockets in the periodontal maintenance phase.

\section{Materials and Methods}

This split-mouth clinical trial was performed on 20 patients (13 females and 7 males with a mean age of $38.3 \pm 1.5$ years) referred to the Periodontics Department of Dental School, Qazvin University of Medical Sciences, and they had recurrent pockets in the periodontal maintenance phase and had previously undergone comprehensive periodontal treatment at least 3 months earlier.

The inclusion criteria were as follows: no systemic diseases, no smoking, not in the pregnancy or breastfeeding period, no regular use of nonsteroidal anti-inflammatory drugs and antibiotics in the last three months, no history of any periodontal treatment for at least the last 3 months, and at least 3 teeth in two different quadrants with 4-7 mm PPD and positive BI. Non-cooperative patients, patients with PI $\geq 1$ and those who had to take antibiotics or antiinflammatory drugs during the study were excluded from the study.

Initially, informed consent forms were obtained from the patients and the study was reviewed and approved by the Ethics Committee of Qazvin University of Medical Sciences (IR.QUMS.REC.1395.310). Furthermore, this study was approved by Iranian Registry of Clinical Trial (identifier: IRCT20201226049831N1).

The patients underwent initial evaluations, and clinical indices, including plaque index (PI), bleeding Index (BI), probing pocket depth (PPD), and clinical attachment level (CAL) were obtained from the marked incisors and premolars. Clinical data were collected from six areas of each tooth by a postgraduate student of periodontics. PPD was measured in millimeters from the free gingival margin to the base of the periodontal pockets using a manual Williams periodontal probe (Hu-Friedy, USA). CAL was also defined as the distance $(\mathrm{mm})$ from the cementoenamel junction (CEJ) to the bottom of the pockets. BI was defined as the presence or absence of bleeding 30 seconds after probing (0.1).$^{20}$ After collecting the indices, the areas in different quadrants with 4-7 $\mathrm{mm}$ PPD and positive BI were randomly divided into experimental (SRP + Laser) and control groups (SRP alone) by throwing coins. Both groups were then matched for PI based on the Silness-Loe plaque index, ${ }^{20}$ and when the treatment of the first area was determined, the next area was treated using another protocol. Then all oral areas were treated with SRP using ultrasonic scalers. Afterwards we used the diode laser in the experimental group to remove the inner layer of the pockets and thus reduce the microbial load. Diode laser (Biolase, Epic10, BIOLASE, Inc, USA) parameters were as follows: wavelength: $940 \mathrm{~nm}$; power: $1 \mathrm{~W}$; 300 $\mu \mathrm{m}$ uninitiated fibers; continuous radiation mode; and energy level: $80 \mathrm{~J} / \mathrm{s}$ (Table 1). The laser fibers then were inserted into the pockets in such a way that they were in contact with the soft tissue wall of the pockets and parallel to the root surface and then they were moved to the apical surface through a horizontal sweeping motion $(1 \mathrm{~mm} / \mathrm{s})$ while remaining in contact with the soft tissue wall of the pockets. The control group underwent treatment using a placebo laser. The areas adjacent to the studied areas were treated with the same protocol. The studied areas did not receive any local anesthesia, and each patient was given oral health training at the same treatment session and examined to re-measure the indices in the first and third months after the treatment.

The data obtained were analyzed by the paired $t$ test and LSD post-test in SPSS version 21 , and $P$ value $<0.05$ was considered statistically significant.

\section{Results}

There were no significant differences in PI, PPD, CAL, and $\mathrm{BI}$ in both groups before the treatment $(P>0.05)$. There were significant improvements in PPD, BI, and CAL in control (SRP) and experimental (SRP + laser) groups one month and three months after the treatment, compared to the pre-treatment phase $(P<0.05)$. (Table 2$)$.

There were also significant improvements in PPD, BI,

Table 1. Laser Parameters

\begin{tabular}{ll}
\hline \multicolumn{2}{l}{ Boilies, Epic 10, BIOLASE, Inc, USA } \\
\hline Wavelength & $940 \mathrm{~nm}$ \\
Power & $1 \mathrm{~W}$ \\
Fiber & $300 \mu \mathrm{m}$ \\
Mode & Continuous mode \\
\hline
\end{tabular}


940-nm Diode Laser Effect on Recurrent Periodontal Pockets

Table 2. Comparing Clinical Indices of Both Groups Before, One and Three Months After Treatment

\begin{tabular}{|c|c|c|c|c|c|c|}
\hline \multirow{2}{*}{ Group } & \multirow{2}{*}{ Index } & Before Treatment & 1 Month After Treatment & 3 Months After Treatment & \multirow{2}{*}{$P$ Value After 1 Month } & \multirow{2}{*}{$\begin{array}{l}P \text { Value After } \\
\text { Months }\end{array}$} \\
\hline & & Mean (SD) & Mean (SD) & Mean (SD) & & \\
\hline \multirow{3}{*}{ SRP + Laser } & PPD & $4.45 \pm 0.38$ & $3 \pm 0.48$ & $2.87 \pm 0.52$ & 0.001 & 0.001 \\
\hline & CAL & $3.59 \pm 1.51$ & $2.38 \pm 1.3$ & $2.32 \pm 1.3$ & 0.008 & 0.001 \\
\hline & $\mathrm{BI}$ & $4.25 \pm 1.5$ & $1 \pm 1.3$ & $0.7 \pm 0.73$ & 0.001 & 0.001 \\
\hline \multirow{3}{*}{ SRP } & PPD & $4.5 \pm 0.29$ & $3.4 \pm 0.5$ & $3.1 \pm 0.48$ & 0.001 & 0.001 \\
\hline & CAL & $3.4 \pm 1.5$ & $2.6 \pm 1.27$ & $2.32 \pm 1.5$ & 0.04 & 0.01 \\
\hline & $\mathrm{BI}$ & $4.15 \pm 2.4$ & $1.9 \pm 1.7$ & $2.1 \pm 1.2$ & 0.001 & 0.002 \\
\hline
\end{tabular}

and CAL in both groups one month after the treatment $(P<0.05)$; however, there were no significant differences in PPD $(P=0.103)$ and CAL $(P=0.012)$ in both groups three months after the treatment. Meanwhile, the improvement in the BI index was significantly higher in the experimental group than in the control group $(P<0.001)$ (Table 3).

\section{Discussion}

The main goal of periodontal treatment is to remove biofilms from the tooth surface. Failure of proper mechanical debridement leads to increased pocket depth and furcation involvement. The complex anatomy of roots and furcations makes it difficult to access subgingival plaques; therefore, it may not be possible to remove microbial plaque completely only by mechanical means, and adjunctive therapies have been proposed to facilitate the plaque removal process. ${ }^{21}$ One of these adjunctive treatments is laser therapy.

Based on the results of the present study, SPR alone and $\mathrm{SPR}+$ Laser resulted in significant improvements in CAL, PPD and BI one and three months after the treatment compared to the pre-treatment phase. There were significant improvements in PPD, CAL, and BI in both groups. In the short term ( 1 month after the treatment), there was a better improvement in PPD, CAL and BI in the experimental group than in the control group, which is consistent with the results of other studies. ${ }^{22-25}$

There were no significant improvements in PPD and CAL in both groups three months after the treatment, but the BI improvement was significantly greater in the experimental group than in the control group, indicating that the SRP + Laser and SRP alone had the same effect on improving PPD and CAL in the long term; however, the SRP+Laser was more effective in improving the BI index than SRP alone. Euzebio Alves et $\mathrm{al}^{26}$ and Nguyen et $\mathrm{al}^{27}$ achieved similar results in their studies, but in a study on the clinical and biochemical effects of diode laser as adjunctive therapy in the treatment of chronic periodontitis, Saglam et $\mathrm{al}^{23}$ showed significant improvements in CAL, PPD, BOP, GI, and PI in the experimental group (SRP + Laser) compared to the control group (SRP alone) after 3 to 6 months of followup.

Most studies have shown that laser treatment leads to improvement in clinical indices in a short-term followup. ${ }^{26-27}$ However, different results have been obtained in a long-term follow-up. Some studies showed no significant difference between diode laser + SRP and SRP alone in the long-term follow-up. ${ }^{19,28}$ However, these two treatments were significantly different only in the BI index in the present study. This discrepancy may be due to several factors, including the different pocket depths, study protocol, and follow-up duration (3 months, 6 months, and 9 months). Other factors such as the laser wavelength, power, radiation duration, and energy level can also be effective in the results of the studies.

Although the use of laser + SRP in most studies did not yield better results than the use of SRP alone in the long term, its benefits in the treatment of periodontal pockets, including bactericidal and curettage effects and its biostimulating properties, cannot be ignored. ${ }^{2}$ Furthermore, the use of laser + mechanical treatments ensures more effective disinfection of the pockets and slower re-

Table 3. Comparing Control and Experimental Groups in Terms of Improvement in the Clinical Indices One and Three Months After Treatment

\begin{tabular}{|c|c|c|c|c|}
\hline \multirow{2}{*}{ Time } & \multirow{2}{*}{ Index } & SRP+Laser & SRP & \multirow{2}{*}{$P$ Value } \\
\hline & & Mean (SD) & Mean (SD) & \\
\hline \multirow{3}{*}{ One month after treatment } & PPD & $3.0 \pm 0.48$ & $3.4 \pm 0.5$ & 0.045 \\
\hline & CAL & $2.38 \pm 1.3$ & $2.6 \pm 1.27$ & 0.023 \\
\hline & $\mathrm{BI}$ & $1 \pm 1.3$ & $1.9 \pm 1.7$ & 0.007 \\
\hline \multirow{3}{*}{ Three months after treatment } & PPD & $2.87 \pm 0.52$ & $3.1 \pm 0.48$ & 0.103 \\
\hline & CAL & $2.32 \pm 1.3$ & $2.32 \pm 1.5$ & 0.12 \\
\hline & $\mathrm{BI}$ & $0.7 \pm 0.73$ & $2.1 \pm 1.2$ & 0.001 \\
\hline
\end{tabular}


colonization compared to mechanical treatments alone..$^{29,30}$ Its curettage property leads to more complete elimination of infected epithelium of the pocket than the use of hand instruments, and without causing any injury to the underlying connective tissue, it reduces the microbial activity of Actinobacillus actinomycetemcomitans and Porphyromonas gingivalis, which are able to penetrate the sulcular epithelium. ${ }^{31}$ Also, the laser treatment of periodontal pockets does not cause pain or discomfort to the patient. ${ }^{32} \mathrm{BI}$ is an important index in assessing the clinical condition of the gingiva. The present study and reviewed articles showed that laser treatment led to a further reduction in this index compared to SRP alone. Although the exact mechanism of this process is not clear, it can be related to reducing the microbial load as well as inflammation and the stimulation of the tissue repair process. ${ }^{33}$ Therefore, some of the advantages of laser treatment in the periodontal maintenance phase include eliminating the need for surgery and especially reoperation, which is important in patients who are not able to undergo surgery due to their systemic conditions. Non-surgical treatment prevents the possible removal of tooth tissue and consequently tooth sensitivity, and the laser treatment also improves the surgery-related dental sensitivity. However, despite all the advantages of the laser, it is still considered as an adjunct therapy in periodontal treatments and cannot replace mechanical treatments. We should not also ignore its disadvantages such as the inability to remove hard sediments, probable thermal damage to hard tissue, higher costs, and longer time required than conventional treatments. In addition, caution should be exercised when using it to prevent visual injuries. In the short term (one month), the SRP + laser was significantly more effective than SRP alone in improving the clinical indices $(P<0.05)$, but the SRP + Laser was more effective than SRP alone only in improving the $\mathrm{BI}$ index in a long-term ( 3 months) followup $(P<0.001)$.

\section{Conclusion}

Both SRP + Laser and SRP alone improved clinical indices in patients. In short-term follow-up, the effect of SRP + Laser treatment on BI, CAL, PPD was significantly superior to SRP treatment alone, but in long-term followup, SRP + laser was more effective than SRP alone in improving the $\mathrm{BI}$ index.

\section{Ethical considerations}

The study was reviewed and approved by the Ethics Committee of Qazvin University of Medical Sciences.

\section{Conflict of Interests}

The authors declare that they have no conflict of interest.

\section{References}

1. Kornman KS. Mapping the pathogenesis of periodontitis: a new look. J Periodontol. 2008;79(8 Suppl):1560-8. doi: 10.1902/jop.2008.080213.

2. Cobb CM. Clinical significance of non-surgical periodontal therapy: an evidence-based perspective of scaling and root planing. J Clin Periodontol. 2002;29 Suppl 2:6-16.

3. Zammit ER. Comparison of Er: YAG laser debridement versus conventional scaling and root planing. Can J Dent Hyg. 2012 ;46(3): 183-186.

4. Nagarakanti S, Gunupati S, Chava VK, Reddy BV. Effectiveness of subgingival irrigation as an adjunct to scaling and root planning in the treatment of chronic periodontitis: a systematic review. J clin diag research. 2015;9(7): ZE06. doi:10.7860/JCDR/2015/13862.6210

5. Kwon T, Lamster IB, Levin L. Current concepts in the management of periodontitis. Int Dent J. 2021; 19:1-15. doi: 10.1111/idj.12630.

6. Tuan MC, Nowzari H, Slots J. Clinical and microbiologic study of periodontal surgery by means of apically positioned flaps with and without osseous recontourning. Int J Periodont Restor Dent .2000;20(5):468-475.

7. Tan AE. Periodontal maintenance. Aus dent J. 2009;54(S1): S110-S117. doi:10.1111/j.1834-7819.2009.01149.x

8. Kaldahl WB, Kalkwarf KL, Patil KD, Molvar MP, Dyer JK. Long-term evaluation of periodontal therapy: I. Response to 4 therapeutic modalities. J Periodontol. 1996;67(2):93102. doi: 10.1902/jop.1996.67.2.93.

9. Mombelli A, Schmid B, Rutar A, Lang NP. Persistence patterns of Porphyromonas gingivalis, Prevotella intermedia/nigrescens, and Actinobacillus actinomyetemcomitans after mechanical therapy of periodontal disease. J Periodontol. 2000;71(1):14-21. doi: 10.1902/jop.2000.71.1.14.

10. Sbordone L, Ramaglia L, Gulletta E, Iacono V. Recolonization of the subgingival microflora after scaling and root planing in human periodontitis. J Periodontol. 1990;61(9):579-84. doi: 10.1902/jop.1990.61.9.579.

11. Kocher T, Fanghänel J, Sawaf H, Litz R. Substance loss caused by scaling with different sonic scaler inserts--an in vitro study. J Clin Periodontol. 2001;28(1):9-15. doi: 10.1034/j.1600-051x.2001.280102.x.

12. Flemmig TF, Petersilka GJ, Mehl A, Hickel R, Klaiber B.The effect of working parameters on root substance removal using a piezoelectric ultrasonic scaler in vitro. $J$ Clin Periodontol .1998 ;25(2):158-63. doi: 10.1111/j.1600051x.1998.tb02422.x.

13. Moëne R, Décaillet F, Andersen E, Mombelli A. Subgingival plaque removal using a new air-polishing device. J Periodontol. 2010 ;81(1):79-88. doi: 10.1902/ jop.2009.090394.

14. Raffetto N. Lasers for initial periodontal therapy. Dent Clin North Am. 2004 ;48(4):923-36, vii. doi: 10.1016/j. cden.2004.05.007.

15. Folwaczny M, Heym R, Mehl A, Hickel R. The effectiveness of InGaAsP diode laser radiation to detect subgingival calculus as compared to an explorer. J Periodontol. 2004 ;75(5):744-9. doi: 10.1902/jop.2004.75.5.744.

16. Romanos GE. Re: Lasers in periodontics: a review of the literature. Cobb CM (2006; 77:545-564). J Periodontol. 2007 ;78(4):595-7; author reply 597-600. doi: 10.1902/ jop.2007.070024. 
17. Crispino A, Figliuzzi MM, Iovane C, Del Giudice T, Lomanno S, Pacifico D, et al. Effectiveness of a diode laser in addition to non-surgical periodontal therapy: study of intervention. Ann Stomatol (Roma). 2015; 18;6(1):15-20.

18. Dederich DN. Little evidence for the use of diode lasers as an adjunct to non-surgical periodontal therapy. Evid Based Dent. 2015;16(1):16. doi: 10.1038/sj.ebd.6401078.

19. Caruso U, Nastri L, Piccolomini R, d'Ercole S, Mazza C, Guida L. Use of diode laser $980 \mathrm{~nm}$ as adjunctive therapy in the treatment of chronic periodontitis. A randomized controlled clinical trial. New Microbiol. 2008 ;31(4):513-8.

20. Newman MG, Takei HH, Klokkevold PR, Carranza FA. Newman and Carranz's Clinical Periodontology. $13^{\text {th }}$ ed. Philadelphia: Elsevier, 2019.

21. Petersilka GJ, Tunkel J, Barakos K, Heinecke A, Häberlein I, Flemmig TF. Subgingival plaque removal at interdental sites using a low-abrasive air polishing powder. J Periodontol. 2003 ;74(3):307-11. doi: 10.1902/jop.2003.74.3.307.

22. Üstün K, Erciyas K, Sezer U, Şenyurt SZ, Gündoğar H, Üstün Ö, et al. Clinical and biochemical effects of 810 $\mathrm{nm}$ diode laser as an adjunct to periodontal therapy: a randomized split-mouth clinical trial. Photomed Laser Surg. 2014 ;32(2):61-6. doi: 10.1089/pho.2013.3506.

23. Saglam M, Kantarci A, Dundar N, Hakki SS. Clinical and biochemical effects of diode laser as an adjunct to nonsurgical treatment of chronic periodontitis: a randomized, controlled clinical trial. Lasers Med Sci. 2014; 29(1):37-46. doi: 10.1007/s10103-012-1230-0.

24. Matarese G, Ramaglia L, Cicciù M, Cordasco G, Isola G. The Effects of Diode Laser Therapy as an Adjunct to Scaling and Root Planing in the Treatment of Aggressive Periodontitis: A 1-Year Randomized Controlled Clinical Trial. Photomed Laser Surg. 2017 ;35(12):702-709. doi: 10.1089/pho.2017.4288.

25. Manjunath S, Singla D, Singh R. Clinical and microbiological evaluation of the synergistic effects of diode laser with nonsurgical periodontal therapy: a randomized clinical trial. J Indian Soc Periodontol. 2020 ;24(2):145-149. doi: 10.4103/jisp.jisp_101_19.
26. Euzebio Alves VT, de Andrade AK, Toaliar JM, Conde MC, Zezell DM, Cai S, et al. Clinical and microbiological evaluation of high intensity diode laser adjutant to nonsurgical periodontal treatment: a 6-month clinical trial. Clin Oral Investig. 2013; 17(1):87-95. doi: 10.1007/s00784012-0703-7.

27. Nguyen NT, Byarlay MR, Reinhardt RA, Marx DB, Meinberg TA, Kaldahl WB. Adjunctive Non-Surgical Therapy of Inflamed Periodontal Pockets During Maintenance Therapy Using Diode Laser: A Randomized Clinical Trial. J Periodontol. 2015; 86(10):1133-40. doi: 10.1902/jop.2015.150152.

28. De Micheli G, de Andrade AK, Alves VT, Seto M, Pannuti CM, Cai S. Efficacy of high intensity diode laser as an adjunct to non-surgical periodontal treatment: a randomized controlled trial. Lasers Med Sci. 2011;26(1):438. doi: 10.1007/s10103-009-0753-5.

29. Gregg RH 2nd, McCarthy DK. Eight-year retrospective review of laser periodontal therapy in private practice. Dent Today. 2003;22(2):74-9.

30. Bach G, Neckel C, Mall C, Krekeler G. Conventional versus laser-assisted therapy of perimplantitis: a five-year comparative study. Implant Dent. 2000;9(3):247-51. doi: 10.1097/00008505-200009030-00010.

31. Romanos GE, Henze M, Baniliashemi S, Parsanejad HR., Winckler J, NentwigGH. Removal of epitheliumin periodontal pockets following diode $(980 \mathrm{mn})$ laser application in the animal model: An in vitro study. Photomed Laser Surg. 2004 ;22(3):177-83. doi: 10.1089/1549541041438597.

32. Passanezi E, Diamante CA, de Rezende ML, Greghi SL. Lasers in periodontal therapy. Periodontol 2000. 2015;67(1):268 -91. doi:10.1111/prd.12067

33. Moritz A, Schoop U, Goharkhay K, Schauer P, Doertbudak O, Wernisch J, et al. Treatment of periodontal pockets with a diode laser. Lasers Surg Med. 1998;22(5):30211. doi: 10.1002/(sici)1096-9101(1998)22:5<302: aid$\operatorname{lsm} 7>3.0 . \operatorname{co} ; 2-\mathrm{t}$. 